\title{
Postreperfusion syndrome, hyperkalemia and machine perfusion in liver transplantation
}

\author{
Damiano Patrono, Renato Romagnoli
}

General Surgery 2U, Liver Transplant Unit, A.O.U. Città della Salute e della Scienza di Torino, University of Turin, Turin, Italy Correspondence to: Renato Romagnoli, MD, FEBS. Associate Professor of Surgery, University of Turin, General Surgery 2U, Liver Transplant Unit, Corso Bramante 88-90, 10126, Turin, Italy. Email: renato.romagnoli@unito.it.

Provenance: This is an invited article commissioned by Academic Editor, Dr. Quan Zhuang (Transplantation Department, The 3rd Xiangya Hospital, Central South University, China Engineering \&Technology Research Center for Transplantation Medicine of National Ministry of Health, Changsha, China).

Comment on: Burlage LC, Hessels L, van Rijn R, et al. Opposite acute potassium and sodium shifts during transplantation of hypothermic machine perfused donor livers. Am J Transplant 2019;19:1061-71.

Received: 21 July 2019; Accepted: 23 August 2019; Published: 11 September 2019.

doi: $10.21037 / \operatorname{tgh} .2019 .08 .12$

View this article at: http://dx.doi.org/10.21037/tgh.2019.08.12

\section{Postreperfusion syndrome in liver transplantation}

In liver transplantation (LT), postreperfusion syndrome (PRS) is represented by a series of transient hemodynamic alterations occurring after graft reperfusion into the recipient, including bradycardia, dysrhythmia, decreased systemic vascular resistance and mean arterial pressure (MAP), and increased pulmonary artery pressure, pulmonary artery wedge pressure and central venous pressure. It can be associated with acute acidosis, hypothermia, hyperkalemia and hyperfibrinolysis. It was first defined by Aggarwal et al. (1) as a decrease in MAP $\geq 30 \%$ from baseline for at least one minute within 5 minutes from graft reperfusion, and further classified by Hilmi et al. (2) into mild PRS (i.e., a $<5$ minutes-long decrease in blood pressure and/ or heart rate $<30 \%$ of the anhepatic levels, responsive to calcium chloride and/or epinephrine intravenous boluses, without continuous vasopressors infusion requirement) and significant PRS (characterized by severe hemodynamic instability with hypotension [ $>30 \%$ of the anhepatic level], asystole, hemodynamically significant arrhythmias, and including also patients with prolonged and/or recurrent fibrinolysis). In severe cases, PRS can degenerate to cardiac arrest and on-table death after graft reperfusion.

Pathogenesis of PRS is complex and incompletely understood. The cause of PRS is generally attributed to the interplay between metabolic acidosis, hyperkalemia, hypocalcemia, hypothermia, air embolism, and the hemodynamic effects of vasoactive substances released at the time of graft reperfusion. After graft reperfusion, many inflammatory cytokines (TNF- $\alpha$, IL-1, IL-2, IL-8) are released into systemic circulation by the grafted liver. Others, like kallikrein, bradykinin, chemokines and activated complement factors are produced by the recipient in response to graft reperfusion. The importance of each of them in determining PRS, however, has not been elucidated so far (3).

Incidence of PRS varies widely across different studies, ranging from $10 \%$ to $58 \%$, and this is at least partially due to some heterogeneity in PRS definition (4-11). Incidence of PRS does not appear to have decreased in recent years; its occurrence is clinically relevant as it has been associated with the onset of postoperative acute kidney injury (AKI) $(12,13)$, early allograft dysfunction, and, although less frequently, reduced graft and patient survival (8).

Risk factors for PRS include donor [age, macrosteatosis, donation after circulatory death (DCD)] and recipient (severity of liver disease, previous myocardial dysfunction) features, and factors related to transplant operation (duration of cold and warm ischemia time, blood products transfusion and calcium requirements, surgical technique, hyperkalemia and hypothermia after graft reperfusion) $(4,5,7,10-12,14)$. The association between factors like donor age, graft macrosteatosis, donation after cardiac death and duration of cold ischemia time suggests that ischemia-reperfusion injury and onset of PRS are closely 
interlinked. In the study by Croome et al. (4), incidence of PRS and on-table cardiac arrest was higher in patients receiving grafts with moderate $(30-60 \%)$ macrosteatosis, as compared to patients transplanted using grafts with mild or no steatosis. Pan et al. (7) observed that matched recipients of grafts from DCD donors, despite having comparable 1-year patient and graft survival rates, had increased rates of PRS (25.7\% versus $12.3 \%)$ and hyperkalemia (33.8\% versus $18.9 \%$ ) as compared to recipients of grafts from brain-dead donors (DBD). The detrimental effects of the combination of macrosteatosis and DCD was shown by Zhang et al. (10), who observed higher rates of PRS and postreperfusion hyperkalemia in recipients of DCD grafts with $\geq 20 \%$ macrosteatosis. Kalisvaart et al. (12) demonstrated the association between PRS, a more severe ischemiareperfusion injury, as reflected by higher transaminases peak, and subsequent AKI. These observations are also in keeping with the role of cold ischemia time as a risk factor for PRS, as prolonged preservation causes progressive adenosine triphosphate (ATP) depletion and aggravates ischemiareperfusion injury (11). Thus, PRS can be interpreted as an early hallmark of severe ischemia-reperfusion injury, explaining its association with $\mathrm{AKI}$ and patient outcome.

\section{Hyperkalemia iN PRS: where does the potassium come from?}

The possibility of PRS and hyperkalemia is well known to anesthetists involved in LT programs. Usually, during the anhepatic phase before graft reperfusion efforts are made to keep potassium levels on the low side, and all means to reduce potassium levels, like sodium bicarbonate, glucose, insulin and calcium infusions, are set ready for use. After graft reperfusion, hyperkalemia can result from acute metabolic acidosis, causing and extracellular potassium ions shift in exchange of $\mathrm{H}^{+}$, from exogenous potassium administration associated with red blood cells transfusion, and from the flush of potassium-rich preservation solution (like University of Wisconsin solution) from the graft into recipient circulation. This last has been particularly emphasized, and some studies have detected an association between potassium concentration of effluent flush fluid and severe PRS (9). However, two facts should be acknowledged. First, liver graft is usually flushed before implantation, either ex-situ with chilled $5 \%$ albumin or saline, or insitu with recipient blood. This practice, which reduces the amount of potassium released by liver graft at reperfusion, has reduced but not eliminated the incidence of PRS.
Second, PRS can present when other preservation solutions with lower potassium concentration are used (Table 1). Thus, it appears that potassium contained in preservation solution is only partially responsible for postreperfusion recipient hyperkalemia.

Intracellular potassium concentration is 140 to $150 \mathrm{mEq} / \mathrm{L}$, and is maintained by the balance between uphill cellular potassium uptake by the activity of sodiumpotassium ATPase, and passive potassium efflux (socalled leak rate) (15). This process requires an intact and functioning cell membrane to actively pump potassium into the cell and limit leak rate. Cold preservation progressively determines cell membrane dysfunction and increased permeability (16) and can therefore reduce sodiumpotassium ATPase activity and increase passive potassium efflux, causing significant release of potassium from the intracellular space. As we would expect that to happen more significantly in more severely damaged liver, recipient hyperkalemia could be interpreted as a further sign of severe ischemia-reperfusion injury. This would provide a rationale to the association between potassium concentration of effluent flush fluid and severity of PRS, and also explain why different potassium concentrations can be measured on graft effluent flush fluid, despite the same preservation solution has been used.

\section{Impact of machine perfusion on PRS}

Since the advent of modern machine perfusion techniques, including normothermic machine perfusion (NMP), hypothermic oxygenated machine perfusion (HOPE) and their variants, a reduction in the incidence of PRS has been a common finding. Angelico et al. (17) compared intraoperative hemodynamics of 6 patients receiving grafts treated with NMP with 12 matched controls preserved by static cold storage (SCS). Although NMP did not significantly reduce raw incidence of PRS $(0 \%$ versus $16.7 \% ; \mathrm{P}=0.53)$, it was associated with higher MAP 90 minutes after graft reperfusion and lower norepinephrine requirement 5, 30 and 60 minutes after graft reperfusion. Furthermore, patients in the SCS group experienced a significant MAP drop 5 minutes postreperfusion, followed by a slow recovery to prereperfusion values after 30 minutes, whereas patients in the NMP group had stable MAP as compared to the anhepatic phase. In the first multicenter randomized trial comparing NMP versus SCS, Nasralla et al. (18) observed, along with a 50\% reduction of ischemia-reperfusion injury in the NMP group (AST peak: 
Table 1 Composition of most common preservation solutions for liver transplantation

\begin{tabular}{|c|c|c|c|c|c|c|}
\hline Solution component or characteristic & Blood & UW & HTK & IGL-1 & SCOT 15 & Celsior \\
\hline Potassium ion (mmol/L) & 5 & 125 & 9 & 25 & 5 & 15 \\
\hline Magnesium ion (mmol/L) & 0.8 & 5 & 4 & 5 & 1.2 & 13 \\
\hline Calcium ion (mmol/L) & 2.5 & & 0.015 & & 1.75 & 0.25 \\
\hline Sulphate ion (mmol/L) & 1.4 & 5 & & & & \\
\hline Phosphate (mmol/L) & 3.2 & 25 & & & & \\
\hline Bicarbonate (mmol/L) & 25 & & & & 25 & \\
\hline Glucose (mmol/L) & 7 & & & & 11 & \\
\hline Adenosine (mmol/L) & & 5 & & 5 & & \\
\hline Histidine (mmol/L) & & & 180 & & & 30 \\
\hline Histidine $\cdot \mathrm{HCl} \cdot \mathrm{H}_{2} \mathrm{O}(\mathrm{mmol} / \mathrm{L})$ & & & 18 & & & \\
\hline Potassium hydrogen 2-ketoglutarate (mmol/L) & & & 1 & & & \\
\hline Mannitol (mmol/L) & & & 30 & & & 60 \\
\hline Glutathione (mmol/L) & & 4 & & 3 & & 3 \\
\hline Allopurinol (mmol/L) & & 1 & & 1 & & \\
\hline Tryptophan (mmol/L) & & & 2 & & & \\
\hline Osmolarity (mOsm) & 308 & 320 & 310 & 290 & 320 & 320 \\
\hline
\end{tabular}

UW, University of Wisconsin; HTK, Histidine-Tryptophan-Ketoglutarate; IGL-1, Institut George Lopez-1; SCOT 15, Solution pour la Conservations des Organes pour la Transplantation-15; HES, hydroxyethyl starch; PEG, polyethylene glycol.

$488 \mathrm{IU} / \mathrm{L}$ versus $965 \mathrm{IU} / \mathrm{L} ; \mathrm{P}<0.001$ ), also a significant reduction of $\mathrm{PRS}$ rate $(12.4 \%$ versus $33 \%$; $\mathrm{P}<0.001)$. The only report of a possible detrimental effect of NMP on PRS was that by Watson et al. (19) from Cambridge. Authors reported 12 cases of LT using declined marginal liver grafts after NMP, among which the first 6 were performed with high mean arterial perfusate $\mathrm{pO}_{2}(621-671 \mathrm{mmHg})$. In this group, 5 patients experienced PRS and 4 had persistent refractory vasoplegia after graft reperfusion, which was referred to high reactive oxygen and nitrogen species production during NMP due to perfusate hyperoxygenation. No case of PRS was observed in the subsequent 6 cases, performed using lower perfusate oxygen tensions $(\sim 150 \mathrm{mmHg})$.

In our experience of hypothermic oxygenated machine perfusion, which has now reached 60 cases, onset of PRS has been anecdotal after HOPE treatment $(20,21)$. In a matched patient cohort, incidence of PRS was reduced in HOPE group (4\% versus $20 \%$; $=0.13$ ), a finding that was confirmed as significant by Bayesian model averaging.

From a mechanistic standpoint, it is likely that 
mitigation of ischemia-reperfusion injury and wash out of inflammatory cytokines have a prominent role in determining the reduction of PRS observed after machine perfusion.

Recently, a paper by Burlage et al. (22) from University Medical Center Groningen, the Netherlands, shed new light on the relationship between machine perfusion, PRS and hyperkalemia. This paper, which analyzes sodium and potassium shifts in liver grafts during hypothermic machine perfusion and subsequent warm reperfusion, was prompted by a previous study from the same group in which, in contrast to what is normally observed at graft reperfusion after SCS, 3 out of 10 LT recipients whose grafts had been treated with HOPE experienced hypokalemia, instead of acute hyperkalemia (23). Briefly, Burlage and colleagues measured sodium and potassium concentrations in the perfusate during 2 hours of dual HOPE (DHOPE, i.e., HOPE performed by simultaneous perfusion of both portal vein and hepatic artery) and in the perfusate of subsequent NMP ( $n=6$, preclinical study) or recipient blood $(n=10$, clinical study), calculating cation shifts during DHOPE, NMP and reperfusion into recipient. The control group was represented by 9 grafts undergoing NMP straight after SCS and by 9 matched patients transplanted with grafts preserved by conventional SCS in the preclinical and clinical study, respectively. In the preclinical study, cation shifts were correlated with markers of liver viability during NMP, including ATP cellular content, perfusate ALT and lactate levels. In the clinical study, the same shifts were correlated with markers of ischemia-reperfusion injury (ALT peak and postoperative day 1 prothrombin time) and with hemodynamic changes in the recipient at graft reperfusion (MAP and norepinephrine requirements). In both the preclinical and clinical study, Authors observed a potassium release in perfusate $(17 \pm 2$ and $34 \pm 6 \mathrm{mmol}$, respectively) and a sodium uptake $(25 \pm 9$ and $24 \pm 14 \mathrm{mmol}$, respectively) during 2 hours of DHOPE. During subsequent NMP, these shifts inverted in DHOPE treated livers, who exhibited active potassium uptake $(19 \pm 3 \mathrm{mmol})$ and sodium release $(7 \pm 3 \mathrm{mmol})$. In contrast, SCS-preserved liver released potassium and absorbed sodium during NMP. In the preclinical study, high potassium perfusate levels during NMP positively correlated with low cellular ATP content, peak ALT and lactate perfusate levels. In the clinical study, blood potassium levels decreased from $4.7 \pm 0.2$ to $3.9 \pm 0.3 \mathrm{mmol} / \mathrm{L}$ after DHOPE, whereas they increased from $4.4 \pm 0.1$ to $5.0 \pm 0.4 \mathrm{mmol} / \mathrm{L}$ in recipients of SCSpreserved livers. Increased potassium levels after portal reperfusion correlated with higher post-LT ALT peak and norepinephrine requirements.

These results are extremely interesting. It is likely that sodium-potassium ATPase dysfunction caused potassium leakage and sodium uptake during DHOPE, which is in keeping with mild edema and weight gain that are commonly observed after HOPE. Upon warm reperfusion, both in the NMP and in-vivo setting, potassium uptake and sodium release by the reperfused liver could be result from preserved cellular membrane integrity and quicker recovery of sodium-potassium ATPase function induced by DHOPE, as opposed to what was observed in livers preserved by SCS. This observation was particularly convincing in the NMP setting, which is a closed circuit allowing avoiding many confounding factors of the in-vivo setting. To this regard, it would have been interesting to have data on potassium level on liver flush fluid obtained before NMP and in-vivo graft reperfusion for both DHOPE and SCS-preserved livers.

It is not surprising that, in the preclinical study, lower cellular ATP levels correlated with higher ALT and lactate perfusate levels and higher potassium levels upon warm reperfusion, as these can all be considered markers of a more severe ischemia-reperfusion injury. Similarly, the association between higher potassium level upon in-vivo graft reperfusion and post-LT ALT peak and norepinephrine requirements go in the same direction.

Overall, the article from the Groningen group is a further proof of the association between ischemia-reperfusion injury, PRS and acute hyperkalemia and provides a new insight on the mechanisms of graft reconditioning and protection during HOPE. PRS and acute hyperkalemia are signs of a more severe graft damage during preservation. Enhanced graft preservation is associated with improved hemodynamic stability upon graft reperfusion and, in some cases, with recipient hypokalemia.

In the setting of NMP, it is likely that enhanced graft and cellular membrane integrity preservation and ATP restoration are also key determinants of reduced PRS rate and improved hemodynamic stability $(17,18)$. A study evaluating sodium and potassium shift during NMP and subsequent in-vivo reperfusion would be particularly interesting.

\section{Clinical implications and future research directions}

The results of Burlage et al. paper have clear clinical implications for anesthesiologic patient management during 
LT. In recipient of HOPE-treated grafts, usual interventions to lower potassium levels could even be harmful and result in acute hypokalemia upon graft reperfusion. More "permissive" potassium levels could therefore be accepted before graft reperfusion.

From another standpoint, these results could move the discussion about the indications for machine perfusion from a donor-oriented to a recipient-oriented approach. As machine perfusion technology comes to a significant cost, many efforts are being made to identify the settings in which it conveys a significant advantage over SCS. So far, these efforts have been focused on donor features. For instance, there is evidence that HOPE is superior to SCS in the setting of DCD LT and graft steatosis $(24,25)$. However, indications for machine perfusion should take into account also recipient features. As suggested by Burlage and colleagues (22), DHOPE treatment could be particularly indicated in patients with pre-LT renal function impairment, as these patients are at increased risk of AKI, which is in turn strongly associated with PRS. Interestingly, in our experience improved postoperative renal function was the main benefit associated with HOPE treatment (21). As for patients at increased risk of AKI, HOPE could be considered in patients with particularly severe liver disease, with pre-LT myocardial dysfunction or when a difficult hepatectomy is foreseen, as these are all risk factors for PRS.

Machine perfusion could also serve as a platform to treat liver graft in an extra-corporeal setting. With regards to PRS, it would be interesting to evaluate the impact of a cytokine filter in parallel to the machine perfusion circuit to decrease the release of inflammatory cytokines at graft reperfusion.

In conclusion, sodium and potassium shifts during DHOPE and warm reperfusion provide further evidence to the concept that PRS and acute hyperkalemia are early signs of severe ischemia-reperfusion injury, which can be mitigated by dynamic preservation techniques. The indications for machine perfusion use could be extended in LT candidates at increased risk of PRS. Future studies evaluating the impact of machine perfusion on PRS should ideally include measurements of cations and cytokines levels on graft effluent flush fluid and recipient blood after graft reperfusion.

\section{Acknowledgments}

None.

\section{Footnote}

Conflicts of Interest: The authors have no conflicts of interest to declare.

Ethical Statement: The authors are accountable for all aspects of the work in ensuring that questions related to the accuracy or integrity of any part of the work are appropriately investigated and resolved.

\section{References}

1. Aggarwal S, Kang Y, Freeman JA, et al. Postreperfusion syndrome: cardiovascular collapse following hepatic reperfusion during liver transplantation. Transplant Proc 1987;19:54-5.

2. Hilmi I, Horton CN, Planinsic RM, et al. The impact of postreperfusion syndrome on short-term patient and liver allograft outcome in patients undergoing orthotopic liver transplantation. Liver Transpl 2008;14:504-8.

3. Jeong SM. Postreperfusion syndrome during liver transplantation. Korean J Anesthesiol 2015;68:527-39.

4. Croome KP, Lee DD, Croome S, et al. The impact of postreperfusion syndrome during liver transplantation using livers with significant macrosteatosis. Am J Transplant 2019;19:2550-9.

5. Fukazawa K, Yamada Y, Gologorsky E, et al. Hemodynamic recovery following postreperfusion syndrome in liver transplantation. J Cardiothorac Vasc Anesth 2014;28:994-1002.

6. Lee J, Yoo YJ, Lee JM, et al. Sevoflurane Versus Desflurane on the Incidence of Postreperfusion Syndrome During Living Donor Liver Transplantation: A Randomized Controlled Trial. Transplantation 2016;100:600-6.

7. Pan X, Apinyachon $W, X i a ~ W$, et al. Perioperative complications in liver transplantation using donation after cardiac death grafts: a propensity-matched study. Liver Transpl 2014;20:823-30.

8. Siniscalchi A, Gamberini L, Bardi T, et al. Post-reperfusion syndrome during orthotopic liver transplantation, which definition best predicts postoperative graft failure and recipient mortality? J Crit Care 2017;41:156-60.

9. Zhang L, Tian M, Sun L, et al. Association Between Flushed Fluid Potassium Concentration and Severe Postreperfusion Syndrome in Deceased Donor Liver Transplantation. Med Sci Monit 2017;23:5158-67.

10. Zhang WJ, Xia WL, Pan HY, et al. Postreperfusion hyperkalemia in liver transplantation using donation 
after cardiac death grafts with pathological changes. Hepatobiliary Pancreat Dis Int 2016;15:487-92.

11. Paugam-Burtz C, Kavafyan J, Merckx P, et al. Postreperfusion syndrome during liver transplantation for cirrhosis: outcome and predictors. Liver Transpl 2009;15:522-9.

12. Kalisvaart M, de Haan JE, Hesselink DA, et al. The postreperfusion syndrome is associated with acute kidney injury following donation after brain death liver transplantation. Transpl Int 2017;30:660-9.

13. Leithead JA, Armstrong MJ, Corbett C, et al. Hepatic ischemia reperfusion injury is associated with acute kidney injury following donation after brain death liver transplantation. Transpl Int 2013;26:1116-25.

14. Zhang L, Tian M, Wei L, et al. Expanded Criteria DonorRelated Hyperkalemia and Postreperfusion Cardiac Arrest During Liver Transplantation: A Case Report and Literature Review. Ann Transplant 2018;23:450-6.

15. Gumz ML, Rabinowitz L, Wingo CS. An Integrated View of Potassium Homeostasis. N Engl J Med 2015;373:1787-8.

16. Huet PM, Nagaoka MR, Desbiens G, et al. Sinusoidal endothelial cell and hepatocyte death following cold ischemia-warm reperfusion of the rat liver. Hepatology 2004;39:1110-9.

17. Angelico R, Perera MT, Ravikumar R, et al. Normothermic Machine Perfusion of Deceased Donor Liver Grafts Is Associated With Improved Postreperfusion Hemodynamics. Transplant Direct 2016;2:e97.

doi: $10.21037 / \operatorname{tgh} .2019 .08 .12$

Cite this article as: Patrono D, Romagnoli R. Postreperfusion syndrome, hyperkalemia and machine perfusion in liver transplantation. Transl Gastroenterol Hepatol 2019;4:68.
18. Nasralla D, Coussios CC, Mergental H, et al. A randomized trial of normothermic preservation in liver transplantation. Nature 2018;557:50-6.

19. Watson CJE, Kosmoliaptsis V, Randle LV, et al. Normothermic Perfusion in the Assessment and Preservation of Declined Livers Before Transplantation: Hyperoxia and Vasoplegia-Important Lessons From the First 12 Cases. Transplantation 2017;101:1084-98.

20. Patrono D, Lavezzo B, Molinaro L, et al. Hypothermic Oxygenated Machine Perfusion for Liver Transplantation: An Initial Experience. Exp Clin Transplant 2018;16:172-6.

21. Patrono D, Surra A, Catalano G, et al. Hypothermic Oxygenated Machine Perfusion of Liver Grafts from Brain-Dead Donors. Sci Rep 2019;9:9337.

22. Burlage LC, Hessels L, van Rijn R, et al. Opposite acute potassium and sodium shifts during transplantation of hypothermic machine perfused donor livers. Am J Transplant 2019;19:1061-71.

23. van Rijn R, Karimian N, Matton APM, et al. Dual hypothermic oxygenated machine perfusion in liver transplants donated after circulatory death. Br J Surg 2017;104:907-17.

24. Kron P, Schlegel A, Mancina L, et al. Hypothermic oxygenated perfusion (HOPE) for fatty liver grafts in rats and humans. J Hepatol 2017. [Epub ahead of print].

25. Schlegel A, Muller X, Kalisvaart M, et al. Outcomes of DCD liver transplantation using organs treated by hypothermic oxygenated perfusion before implantation. J Hepatol 2019;70:50-7. 